\title{
Global changes of CR geomagnetic cutoff rigidities and two- parametric representation of long-term variation spectra
}

\section{L.I. Dorman ${ }^{1,2}$}

1. Pushkov's IZMIRAN, Russian Academy of Science, Moscow, Russia;

2. Israel Cosmic Ray \& Space Weather Center and Emilio Segre' Observatory, affiliated to Tel Aviv

University, Shamir Research Institute, and Israel Space Agency, Israel;

lid010529@gmail.com

P.G. Kobelev ${ }^{1}$

1. Pushkov's IZMIRAN, Russian Academy of Science, Moscow, Russia; kosmos061986@yandex.ru

V.G. Yanke ${ }^{1}$

1. Pushkov's IZMIRAN, Russian Academy of Science, Moscow, Russia; yanke@izmiran.ru

Abstract. The values for two-parametric variations spectra are determined for the whole period of observations in 1957-2017 years. A long-period changes of spectra with taking into account of cut-off rigidity changes are presented.

Keywords: cosmic rays, variation spectra, geomagnetic cutoff rigidity.

\section{Introduction}

Since the mid-1950s, the world network of neutron monitors, muon telescopes and stratospheric sounding detectors has been used to study the physical processes in the interplanetary medium and to determine the structure of the Heliosphere. The relative secondary variations in the counting rate of the detectors, which in the approximation of zero harmonics are related to the spectrum of primary variations of cosmic rays reflecting the Fredholm integral equations of the first kind, are subject to measurement:

$$
v^{i}=\int_{R_{c}^{i}}^{\infty} W^{i}\left(R_{c}^{i}, h_{0}^{i}, R\right) \frac{\partial J}{J}(R) d R,
$$

where the connection function $W^{i}$ (coupling function according to Dorman, 1957) acts as the core of the integral equation, and the spectrum of variations $d J / J$ acts as the unknown function. In our case, an important circumstance is that the desired solution can be sought as an analytical function of the spectrum of variations $d J / J$ with a certain number of parameters.

\section{Method and Experimental Data}

The spectrum of cosmic ray variations can be represented in two-parameter form

$$
\partial J / J(R)=a_{1} R^{-\gamma},
$$

where $a_{1}$ is a variation of the flow of particles with a hardness of $1 \mathrm{GV}$, and $\gamma>0$ is a parameter of the power spectrum. It is preferable to use the spectrum of variations given for $10 \mathrm{GV}$ species.

$$
\partial J / J(R)=a_{10}\left(\frac{R}{10}\right)^{-\gamma},
$$

where $a_{10}=a_{1} 10^{-\gamma}$ is the amplitude of particles with a rigidity of $10 \mathrm{GV}$, which is close to the effective rigidity for neutron monitors. 
The system of equations (1) can be linearized by decomposing into a Taylor series in the neighborhood of the zero approximation. Obtained equations of the form

$$
\left.v^{i} \approx v^{i}\right|_{0}+\left.A_{1}^{i}\right|_{0}\left(a-a^{0}\right)+\left.A_{2}^{i}\right|_{0}\left(\gamma-\gamma^{0}\right)
$$

are solved by the method of successive approximations. The decomposition coefficients $A_{1}$ and $A_{2}$ for the spectrum reduced to $10 \mathrm{GV}$ are defined as

$$
A_{1}^{i}=C_{00}(\gamma), A_{2}^{i}=a_{10} \int_{R_{c}^{i}}^{\infty} \ln \left(\frac{R}{10}\right) \Psi^{i}(\gamma, R) d R \text {, where } \Psi^{i}(\gamma, R)=W^{i}\left(R_{c}^{i}, h_{0}^{i}, R\right)\left(\frac{R}{10}\right)^{-\gamma}
$$

To solve the system of equations (4), it is required to perform a certain number of iteration steps from the point of zero approximation. Then, for determining the reception coefficients of the zero harmonic of the neutron, muon, and charged components in the atmosphere, we used communication (coupling) functions from Aleksanyan et al. (1982), Fujimoto et al. (1977), and Svirzhevsky (2002). To take into account longterm changes in geomagnetic cutoff rigidity for the period under study, the results of the work were used (Gvozdevsky et al., 2019). For each month, a graphical result is formed, an example of which is shown in Figure 1.
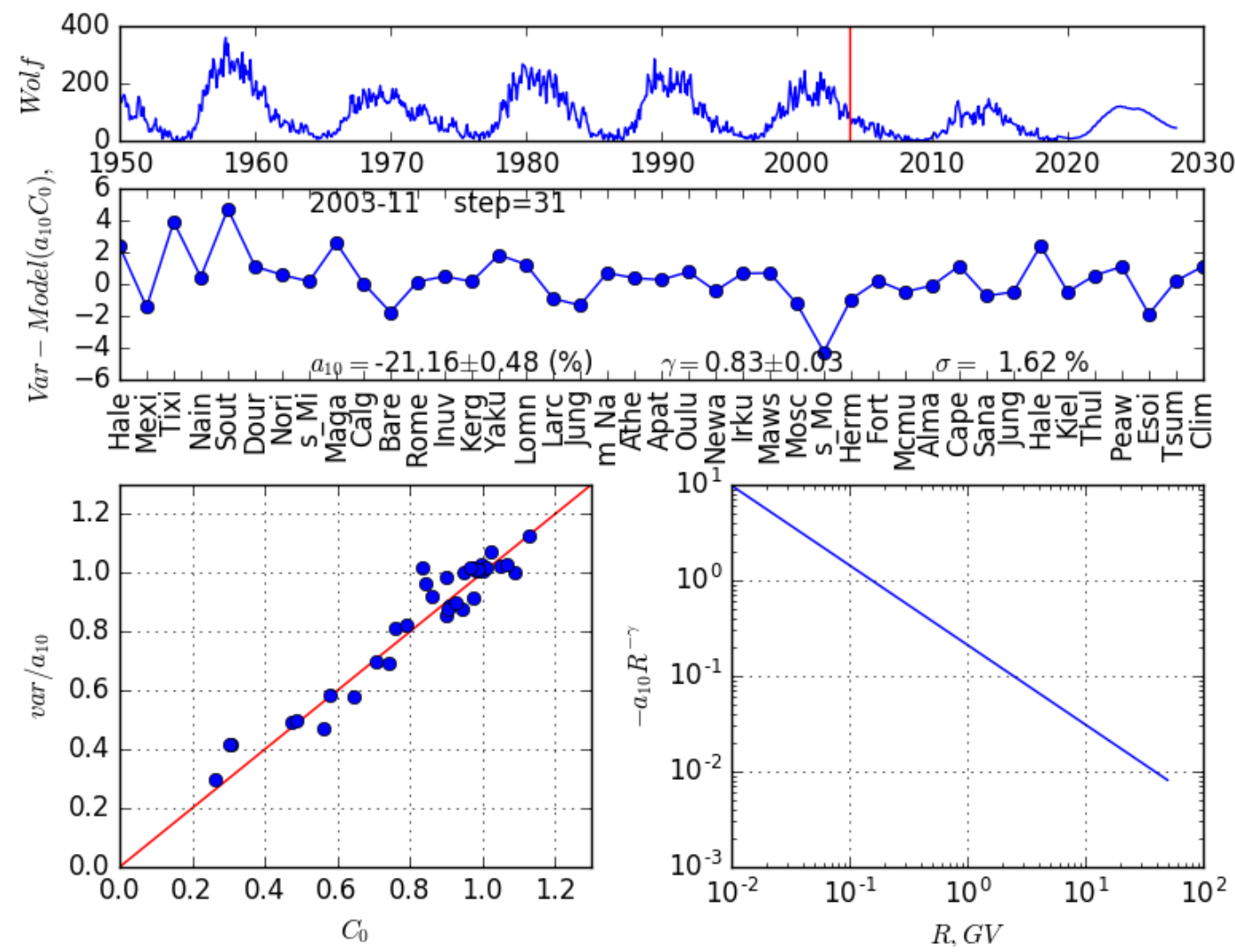

Figure 1. Sample graphical result for November 2003

In Figure 1 on the second panel above shows the discrepancy between the observed variations of all the detectors and the model for the point in time, marked as a vertical line on the top panel on the Wolf number curve. The residuals panel also shows the found parameters of the $a_{10}$ and $\gamma$ variation spectrum for a given point in time. The lower left panel shows the dependence of variations normalized to $a_{10}$ on the receiving coefficients $C_{0}$. The first point in the region of small $C_{0}$ refers to the vertical component of the Nagoya muon telescope, the last point in the region of large $C_{0}$ refers to the detector of the stratospheric sounding, the remaining points to the neutron monitors.

Of course, a good correlation ( $>0.9$ ) is observed only for the maximum of solar activity, when large variations are observed relative to the base period. The desired stiffness spectrum of $d J / J$ variations in the double logarithmic scale is shown in the lower right panel. 
In Fifure 2 on the right panel shows the convergence of the method in the minimum (1996) and maximum (1992) of solar activity. The linear parameter $a_{10}$ convergence is very fast. From the starting point X0, the solution for $a_{10}$ is determined almost in one iteration, and then there is a slow search for a solution for $\gamma$ with a refinement of $a_{10}$. The number of iterations can reach several dozen. In the upper right part of the figure for 1987 (a sharp minimum with a very small difference in values from 2009), an example of a divergent solution is given; in this case, the problem converges only with small $(\sim 0.4)$ starting values of $\gamma$. On the right panel of Fifure 2 given a geometric interpretation of convergence.
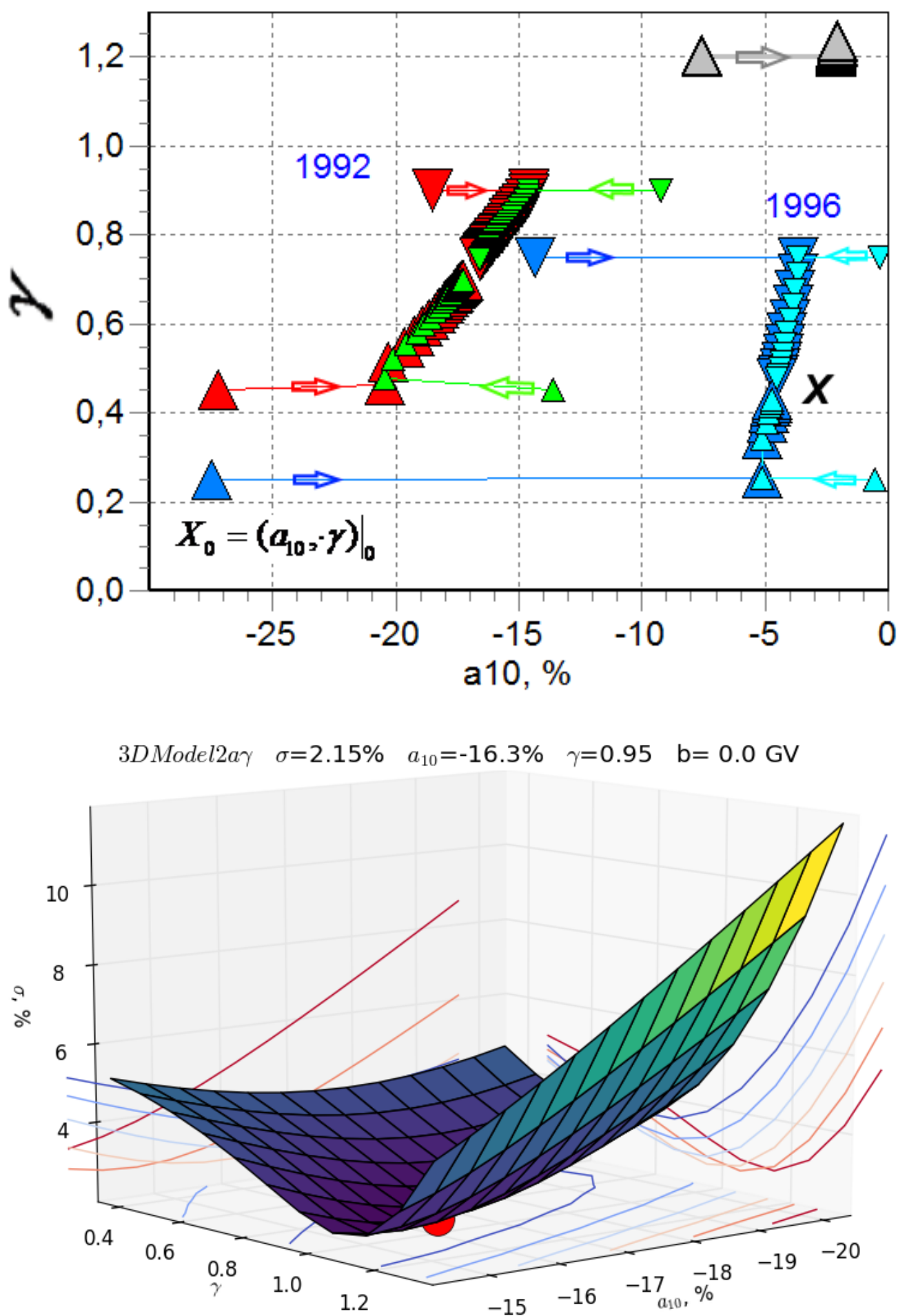

Figure 2. Illustration of the convergence method for the two-parameter spectrum of variations.

In this paper, several new points were taken into account: a) the data series under consideration was supplemented with data from the last decade, $b$ ) analysis of the spectrum of variations was carried out for the 2009 base, which improved the accuracy of the determination of spectrum parameters, $c$ ) the $l t v-d b$ 
database was developed to solve the problem, $d$ ) changes in geomagnetic cutoff rigidity were taken into account (Gvosdevsky et al., 2016, 2017) for the network of cosmic ray stations for the period under consideration.

\section{Calculation results and their analysis}

In Figure 3 for the entire period under consideration, long-term changes in the parameters of the spectrum of variations and some characteristics of a system of linear equations are shown.

The first and second panels show the time dependences of the amplitude of the spectrum of variations a10 and the exponent $\gamma$ of the spectrum with errors in their determination. The comparison shows good agreement with the results of [Yanke et al., 2019]. The third panel shows the time dependence of the root-mean-square error of the distribution of the residuals of the system of equations.

The fourth panel shows the condition numbers of a system of equations that indicate a good convergence of the solution for all periods except the base one. The last panel shows the number of iterations for each point in time. To speed up the process of convergence, the previous solution was used as the initial approximation, which made it possible to halve the solution time.
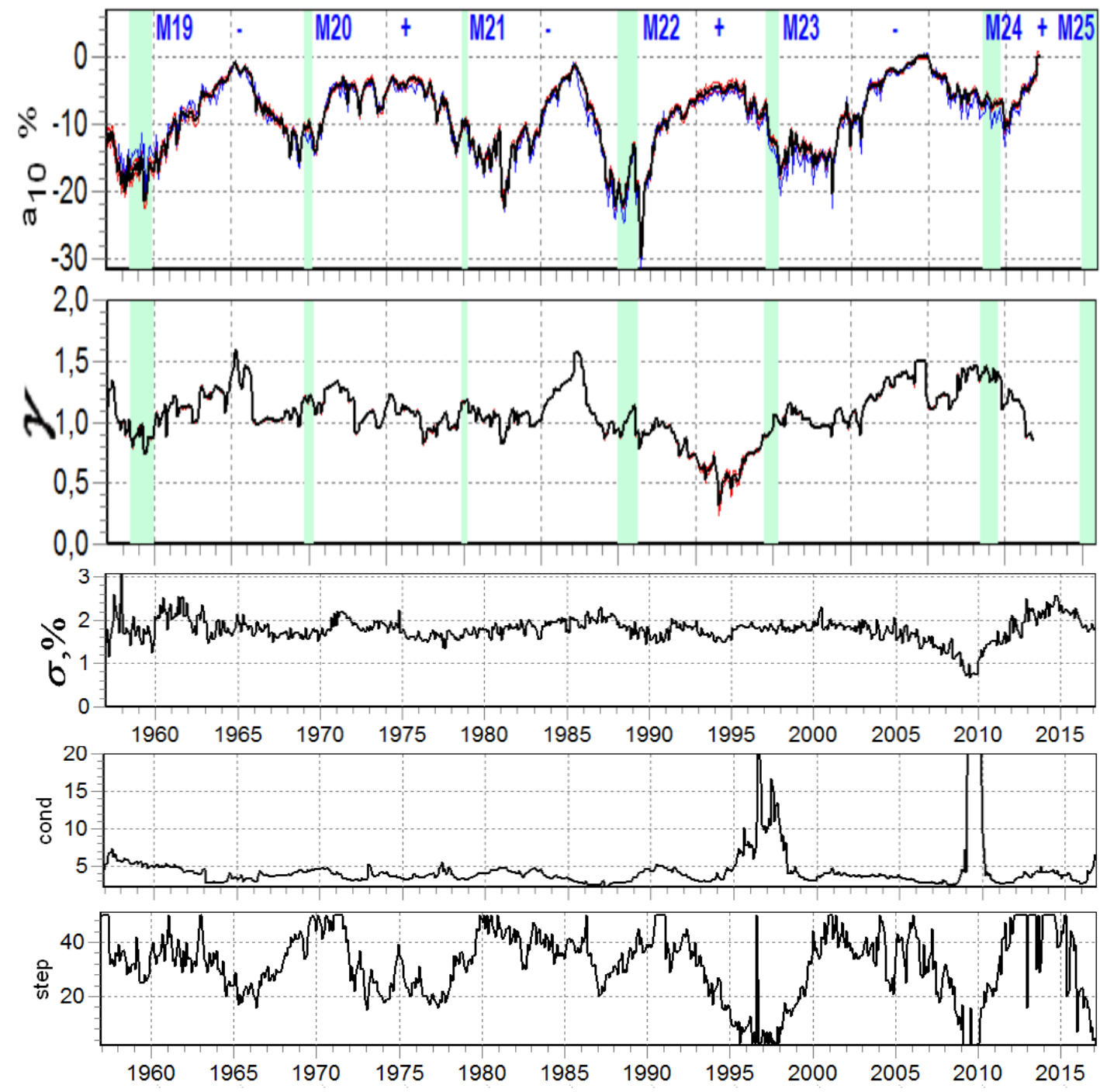

Figure 3. Long-term changes in the parameters of the spectrum of variations and characteristics of the system of equations for the entire period under consideration.

\section{Conclusion}

The proposed method of solving the system of equations by the linearization method has shown high efficiency with the possibility of quantitative assessment of the parameter values, their correlations and errors. Although a purely power spectrum of variations is a simple approximation, the results are in good agreement with the conclusions of other works [Yanke et al., 2019]. All obtained numerical and graphical results can be found in the archive [LTV, 2019]. Further work should be carried out in the direction of increasing the number of spectrum parameters for a more accurate description in the lower stringency 
region.

The analysis showed that the uncertainty of the desired spectrum of variations due to the global change in the geomagnetic cutoff rigidity is insignificant, since there are practically no cosmic ray detectors in the region of the North and South Atlantic anomalies.

Acknowledgements: This work was supported by the RFBR grant 17-02-00508a. The work is based on the experimental data in the frame of the Project "Russian National Network of Cosmic Ray Stations" and the World Network of Cosmic Ray Stations (http://www.nmdb.eu)

\section{References}

Aleksanyan T.M., Belov A.V., Yanke V.G. et al., 1982. Experimental Studies of Geomagnetic Effects in Cosmic Rays and the Spectrum of the Increasing Effect Before Magnetic Storms, Izv. RAS. Ser. Phys., vol. 46, No. 9, p. 1668-1691.

Dorman L.I., 1957. Cosmic Ray Variations, Gostekhteorizdat, Moscow. In Russian. Translation in English: Department of Defense, USA (1958).

Fujimoto K., Murakami K., Kondo I., and Nagashima K., 1977. Approximate formula for response function of cosmic ray hard component at various depths of the atmospheres and underground // Proc 15-th ICRC, Plovdiv, Bulgaria., V.4, 321-325.

Gvozdevsky B.B., Belov A.V., Gushchina R.T., Eroshenko E.A., and Yanke V.G. 2019. Planetary long term changes of the cosmic ray geomagnetic cut off rigidities, J. Phys.: Conf. Ser. 1181 012008, 2019.doi: $10.1088 / 1742-6596 / 1181 / 1 / 012008$.

LTV, 2019. Long Time Variation [Electronic resource], ftp://crsb.izmiran.ru/LTV/2019.

Svirzhevsky N.S., 2002. Thesis, FIAN.

Yanke V.G., Belov A.V., Gushchina R.T.,and Zirakashvili V.N., 2019. The rigidity spectrum of the longterm cosmic ray variations during solar activity cycles 19-24, J. Phys.: Conf. Ser. 118112007. doi:10.1088/1742-6596/1181/1/012007. 